\title{
Comparative study of fungal stability between Metarhizium strains after successive subculture
}

\author{
Rana H. M. Hussien ${ }^{1,2^{*}}$ (D), Said M. Ezzat ${ }^{1}$, Ali A. El Sheikh², James W. D. Taylor ${ }^{3}$ and Tariq M. Butt ${ }^{3}$
}

\begin{abstract}
Background: Metarhizium species are considered one of the most outstanding powerful biological control agents that have been commercialized as biopesticides against various agricultural pests. Fungal stability with successive in vitro cultivation is a desirable trait for a large-scale production of fungal biopesticide.

Main body: The new Egyptian strain Metarhizium anisopliae AUMC 3262 exhibited auspicious results when compared to Metarhizium brunneum ARSEF 4556 and M. brunneum V275 based on the variations of fungal characteristics, and essential quality control parameters (radial growth rate, conidial yield, viability, and virulence) after repeated in vitro subculturing. Changes in morphological characteristics were noted at both AUMC 3262 and ARSEF 4556. Following the 5th subculture, decreased conidial yield was noted, though radial growth remained stable, confirming that there is a non-positive correlation between conidial yield and radial growth rate for these species. In contrast, V275 showed a high morphological stability, conidial yield, and radial growth rate after repeated subculture. The three tested strains manifested high viability up to $100 \%$ and displayed the same pattern of $\operatorname{Pr} 1$ production. A slight variation was recorded in the median lethal time $\left(L T_{50}\right)$ values against the great wax moth, Galleria mellonella (L.), larvae between different subcultures of the tested Metarhizium strains.
\end{abstract}

Conclusion: The new Egyptian strain AUMC 3262 showed a high stability with a slight difference in some parameters after the successive subculture compared to both ARSEF4556 and V275.

Keywords: Fungal stability, Metarhizium strains, Pr1, Virulence

\section{Background}

Application of the entomopathogenic fungi (EPF), as an alternative to conventional chemical pesticides, represents a safe sustainable crop protection method for integrated pest management (IPM) strategies. More than 700 species of EPF are used as biological control agents, with particular emphasis on Metarhizium spp. (Humber, 2008). Approximately $39 \%$ of mycoinsecticides based on genus Metarhizium as an active ingredient (Faria and Wriaght, 2007), probably due to their high host

\footnotetext{
* Correspondence: ranahussien46@gmail.com

'Department of Botany, Faculty of Science, Zagazig University, Zagazig, Egypt ${ }^{2}$ Department of Pest Physiology, Plant Protection Research Institute,

Agricultural Research Center, Giza, Egypt

Full list of author information is available at the end of the article
}

specificity, non-persistence, and eco-friendly with a unique mode of action against target pests. M. anisopliae showed high mortality rates against the stored grain pests Sitophilus granarius and S. oryza depending on conidial concentrations and the absence or presence of food (Ak, 2019), while evaluation of different strains of M. anisopliae against Spodoptera exigua and S. littoralis showed a high accumulative mortality rate (100\%) against 2nd and 4th larval instars of S. littoralis and 1st and 3rd instars of S. exigua larvae (Anand and Tiwary, 2009; Han et al., 2014).

EPF used as biological control agents are characterized by critical factors to be used effectively in IPM programs, one key aspect concerning propagule stability after repetitive subculture or mass production. Genus

\section{Springer Open}

๑ The Author(s). 2021 Open Access This article is licensed under a Creative Commons Attribution 4.0 International License which permits use, sharing, adaptation, distribution and reproduction in any medium or format, as long as you give appropriate credit to the original author(s) and the source, provide a link to the Creative Commons licence, and indicate if changes were made. The images or other third party material in this article are included in the article's Creative Commons licence, unless indicated otherwise in a credit line to the material. If material is not included in the article's Creative Commons licence and your intended use is not permitted by statutory regulation or exceeds the permitted use, you will need to obtain permission directly from the copyright holder. To view a copy of this licence, visit http://creativecommons.org/licenses/by/4.0/. 
Metarhizium has the ability to be mass produced using a biphasic production system on a wide variety of relatively inexpensive organic substrates (Jenkins et al., 1998). Moreover, M. anisopliae AUMC 3262 could be mass produced on cheap agriculture byproducts using biphasic production technique (Ezzat et al., 2019).

Sector formation is an important sign of fungal instability resulting from cultural degeneration. Sectors can differ from the parent culture in a range of morphological and physiological characteristics, including a decline in infective propagule production and certain metabolites, resulting in decreased fungal pathogenicity (Shah and Butt, 2005). The sterile sectors cause reduced spore yield and increased production costs, making the end product less cost-effective; in addition, a virulent inoculum could decrease the end product efficacy when compared with competing agents (Rayan et al., 2002).

The production of stable conidial yield with high viability is also an imperative parameter for commercial purposes as insect mortality is dose-dependent; the more conidia that adhere to the body of the insect, the faster the fungus will kill the host insect (Butt, 2002). Following conidial attachment to the insect cuticle, the conidia germinate and exert a combination of passive hydrophobic and electrostatic forces, followed by secretion of cuticle-degrading enzymes. Cell wall-bound subtilisin protease Pr1 plays a pivotal role in insect cuticle penetration. Furthermore, $\operatorname{Pr} 1$ is classified as a pathogenicity determinant (St. Leger et al., 1987). Small and Bidochka (2005) found that M. anisopliae Pr1 genes are upregulated from the point of initial infection until the mycelia emerge and produce conidia on the surface of the cadaver, indicating a potential link between conidial virulence and $\operatorname{Pr} 1$ production. Consequently, the ability of $M$. anisopliae conidia to produce a high amount of Pr1 qualifies its use as a biocontrol agent.

Virulence maintained over several generations is the most important factor in choosing the fungi as effective biocontrol agents. Several reports have stated that EPF, including $M$. anisopliae, tended to experience a loss or reduction in sporulation and virulence (attenuation), following successive subculture on artificial media (Santoro et al., 2014). These studies also confirmed that the virulence was improved or restored when the pathogen was passed through a suitable insect host or particular culture media (Wang and St. Leger, 2005).

In this study, a new Egyptian isolate, $M$. anisopliae AUMC 3262, the promising M. brunneum ARSEF 4556, and the commercial $M$. brunneum V275 strains were evaluated for their fungal stability and efficacy that could help in developing mass production strategy and commercialization process.

\section{Main text \\ Materials and methods \\ Fungal isolates}

The Metarhizium strains used in this comparison study were M. anisopliae AUMC 3262 isolated from the great wax moth, Galleria mellonella L. (Lepidoptera: Pyralidae), Egypt; M. brunneum ARSEF 4556 isolated from Boophilus sp. (Acari: Ixodidae), the USA; and M. brunneum V275 isolated from Cydia Pomonella L. (Lepidoptera: Torticidae), Austria. Prior to their use in these studies, all 3 fungal strains were first passed through the insect host, G. mellonella, in order to restore virulence, before being re-isolated on Sabouraud dextrose yeast agar (SDAY), composed of $40 \mathrm{~g}$ glucose, 10 neopeptone, 2 yeast extract, and 15 agar in 11 dist. $\mathrm{H}_{2} \mathrm{O}$ (Sigma-Aldrich, UK) incubated in total darkness at $25 \pm 2{ }^{\circ} \mathrm{C}$ for 15 days. Subcultures were maintained on SDAY at $4{ }^{\circ} \mathrm{C}$ for subsequent studies.

\section{Insect rearing}

Galleria mellonella cultures were maintained in 2-1 glass jars covered with a muslin cloth at $27 \pm 2{ }^{\circ} \mathrm{C}$ and 8:16 (L:D) $\mathrm{h}$ photoperiod, provided with honey and wheat germ as nutrient. All individuals used in this study were last larval instars, with the size range of $15-25 \mathrm{~mm}$.

\section{Effect of successive subculture on quality control parameters}

Fungal plugs $(8 \mathrm{~mm})$ were taken from the edge of the colony of a 15-day-old culture and used to inoculate Petri dishes of SDAY incubated at $25 \pm 2{ }^{\circ} \mathrm{C}$ for 7 days. This culture represented the first subculture. Successive subcultures were prepared by inoculating fresh SDAY plates with an inoculum of the previous subculture, with each subculture grown on SDAY for 7 days at $25 \pm 2{ }^{\circ} \mathrm{C}$. Following incubation, subcultures were stored at $4{ }^{\circ} \mathrm{C}$ until the 9th subculture had been produced. After the production of the 9th subculture, 1st, 3rd, 5th, 7th, and 9th subcultures were grown on new SDAY for 7 days. This procedure allowed the simultaneous generation of these subcultures under similar growth conditions (5 replicates per subculture). These cultures were then used for subsequent studies.

\section{Monitoring fungal growth and sector formation}

After the simultaneous growth, conidia of 7-day-old cultures were scraped from the simultaneously cultivated plates, spread on to $90 \mathrm{~mm}$ SDAY Petri dish, and incubated in darkness for 3 days at $25 \pm 2{ }^{\circ} \mathrm{C}$. Individual plugs ( $8 \mathrm{~mm}$ diameter) of un-sporulated mycelium from the 3-day-old cultures were placed upside down in the center of SDAY medium in 90-mm Petri dishes and incubated in darkness for 15 days at $25 \pm 2{ }^{\circ} \mathrm{C}$. Three replicates were prepared from each replicate of the five 
replicates previously prepared (total no. 15). Morphological changes and sector formation were observed daily, and surface radial growth was recorded from the 3rd to 15th days and was calculated following the equation:

Colony diameter at the end of incubation period - Fungal disc diameter Total incubation days

\section{Conidial yield and viability}

From the previous prepared 15 plates, 5-mm agar plugs were taken randomly after 15-day incubation from each plate and placed in $1 \mathrm{ml}$ of $0.03 \%(\mathrm{v} / \mathrm{v})$, Tween 80 then vortexed to suspend the spores. Spore yield was determined, using an Improved Neubauer hemocytometer, Germany. Germination and viability were assessed according to Inglis et al. (2012). 15 SDAY plates were inoculated with $50 \mu$ of the previous conidial suspension $\left(4 \times 10^{7}\right.$ conidia/ml), then incubated in darkness at $25 \pm 2{ }^{\circ} \mathrm{C}$, after $20-24 \mathrm{~h}$. Plates were examined for germination at $\times 40$ (100 conidia per microscopic field). A spore was considered to have germinated if it had formed a germ tube that was as long as the spore width.

\section{Determination of spore bound Pr1}

Conidia-bound Pr1 activity was determined according to Shah et al. (2005) as follows: $10 \mathrm{mg}$ of conidia was harvested from 15-day-old culture and washed once with $1 \mathrm{ml}$ of $0.03 \%(\mathrm{v} / \mathrm{v})$ aq. Tween 80 and twice with $1 \mathrm{ml}$ distilled water, then incubated in $1 \mathrm{ml}$ of $0.1 \mathrm{M}$ Tris- $\mathrm{HCl}$ (pH 7.95) containing $1 \mathrm{mM}$ Succinyl-AlaAla-Pro-Phe-p-nitroanilide (Sigma-Aldrich UK) for 5 $\mathrm{min}$ at room temperature. After incubation, conidia were pelleted by centrifugation at $12,000 \mathrm{rpm}$ for 5 min (Sanyo, Harrier 18/80 centrifuge). The supernatant $(200 \mu \mathrm{l})$ was transferred to a 96-well flatbottom plate, optical grade (Greiner Bio-One) and absorbance measured, using a BioTek Synergy H1 multi-mode reader at $405 \mathrm{~nm}$. Buffered substrate was used as a control.

\section{Determination of fungal virulence}

Assessment of conidial virulence for each subculture was carried out against the last larval instar of $G$. mellonella. Batches of $10 \mathrm{G}$. mellonella larvae were immersed for $10 \mathrm{~s}$ in $10 \mathrm{ml}$ of $0.03 \%(\mathrm{v} / \mathrm{v})$ aqueous Tween 80 conidial suspension $\left(4 \times 10^{7}\right.$ conidia/ml $)$ prepared from each subculture. Excess conidial suspension was removed by transferring the inoculated insects to filter paper before moving them to a 90$\mathrm{mm}$ Petri dish lined with filter paper moistened with distilled water and sealed with paradigm tape. No food was provided. Control insects were immersed in
$0.03 \%(\mathrm{v} / \mathrm{v})$ aqueous Tween 80 only. Treatments were carried out in triplicate. All dishes were incubated at $25 \pm 2{ }^{\circ} \mathrm{C}$ in darkness and monitored daily for 5 days.

\section{Statistical analysis}

$\mathrm{LT}_{50}$ was calculated using Bio-Stat V5 (analyst soft inc. V.5.9.33). Other results were expressed as the mean \pm standard error (SE). Statistical significance was determined by the way of analysis of variance (one-way ANOVA, SPSS software version 16), followed by the least significant difference LSD test.

\section{Results and discussion}

Effect of successive subculture on quality control parameters

\section{Morphological characteristics}

Specific criteria must be met by EPF to be used as biocontrol alternatives to chemical pesticides. Sector formation and changes in morphological and physiological characteristics could cause significant commercial problems including a decline in the production of certain metabolites and virulence as well as conidial yield. All of the tested strains shared almost the same morphological appearance of a circular, dark olivaceous-green colony. ARSEF 4556 was characterized by a very good sporulation pattern with the 1st subculture, as the conidia were arranged as central layers. Following the 5th subculture, yellow sectors appeared and increased gradually in size with the following subcultures, while AUMC 3262 was found to have small, central white mycelial growth, which increased in size from the 7th subculture until full coverage of the Petri dish was observed at the 9th subculture. The changes in fungal morphology and sector appearance were noted in both AUMC 3262 and ARSEF 4556 after the 5th and 7th subcultures and increased with the successive subcultures were often observed in fungal cultures maintained on artificial media as a result of cultural degradation, caused by age of the culture, method of propagation or nature of the culture media itself (Shah and Butt 2005 and Wang et al., 2005a). This is attributed to mutation, transposons, or genomic rearrangement (Firon et al., 2002). In contrast, V275 maintained its morphology until the 9th subculture. A small mycelial growth appeared over the colony center (Fig. 1).

\section{Monitoring of radial growth rate, conidial yield, and viability}

Effective EPF must be characterized by the production of a highly stable conidial yield with high viability along with frequent subcultures. The growth rate and conidial yield varied across the tested Metarhizium isolates, and the radial growth rate of AUMC 3262 was found to be 

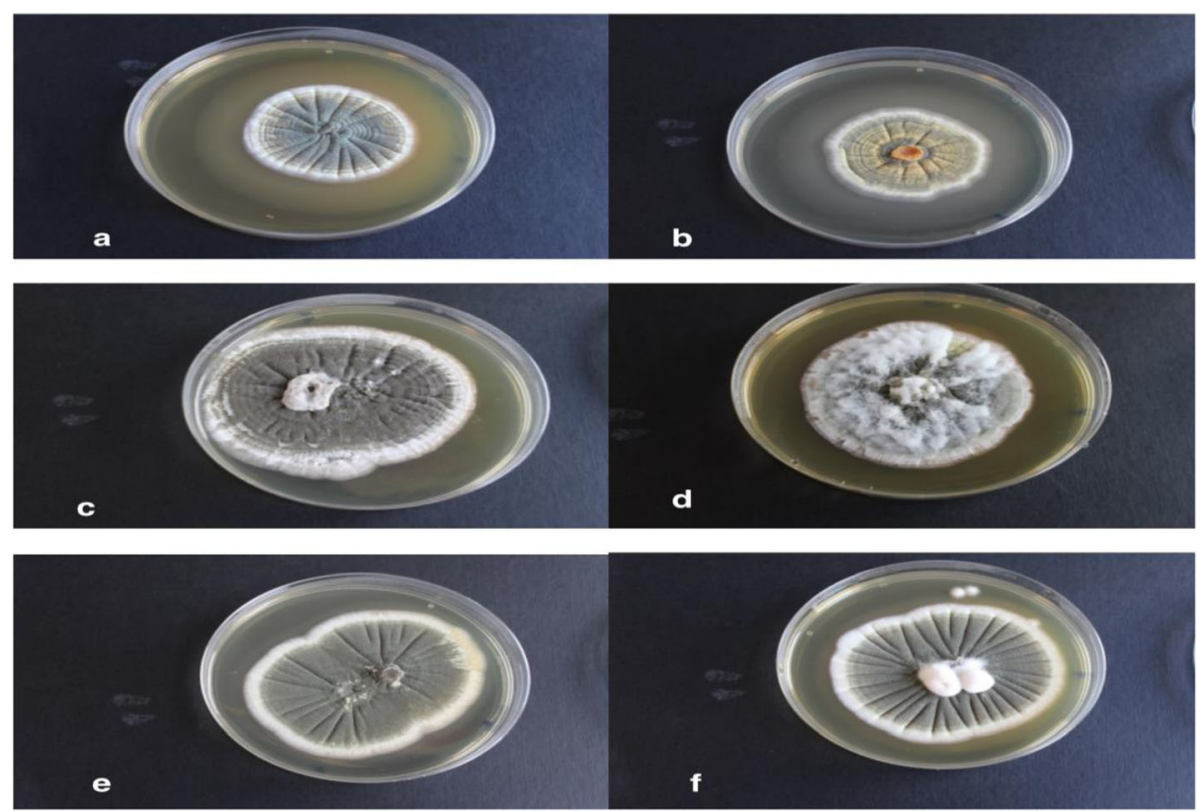

Fig. 1 Influence of successive subculture on morphology and sector formation after 15 days growth on SDAY at $25 \pm 2{ }^{\circ} \mathrm{C}$. a The first subculture of ARSEF 4556. b The ninth subculture of ARSEF 4556. c The first subculture of AUMC 3262. $\mathbf{d}$ The ninth subculture of AUMC 3262. e The first subculture of V275. $\mathbf{f}$ The ninth subculture of V275

different between different subcultures, gradually decreasing in size from $4.2 \pm 0.11 \mathrm{~mm} /$ day at 1 st subculture to $3.7 \pm 0.13 \mathrm{~mm} /$ day at 9 th subculture. Furthermore, the conidial yield obtained with AUMC 3262 showed some instability, ranging from $4.43 \pm 0.69 \times 10^{7}$ conidia $/ \mathrm{ml}$ at the 1 st subculture to $1.93 \pm 0.40 \times 10^{7}$ conidia/ml at 5 th subculture, which was found to be the lowest conidial yield observed. On the other hand, V275 and ARSEF 4556 offered a relatively stable radial growth pattern between subcultures, ranging from $3.4 \pm 0.54$ to $3.9 \pm 0.11 \mathrm{~mm} /$ day for V275 and $2.75 \pm 0.08$ to $2.60 \pm$ $0.06 \mathrm{~mm} /$ day for ARSEF 4556, which represent the lowest linear growth rate through different subcultures. Although the 1st subculture of ARSEF 4556 showed a superior production of conidia with a value of $6.23 \pm$ $0.23 \times 10^{7}$ conidia $/ \mathrm{ml}$, a significant decrease $(P<0.05)$ in conidial yield observed through successive subculture, dropped to $3.36 \pm 0.18 \times 10^{7}$ conidia/ml at 9 th subculture, whereas V275 displayed a high stability in conidia production through successive subcultures, ranging from $4.46 \pm 0.38$ to $3.80 \pm 0.55 \times 10^{7}$ conidia $/ \mathrm{ml}$ with a nonsignificant difference through successive subcultures $(P$ $>0.05$ ) as shown in Table 1 . This study revealed that the radial growth rate varied between fungal species and strains, with no relationship between conidial yield and radial growth rate. Similarly, Safavi et al. (2007) reported that $M$. anisopliae strains showed a high radial growth rate with a low conidial yield in contrast to 3 tested isolates of $B$. bassiana, which provided a high radial growth rate with a high conidial yield, depending on the provided nutrition. This suggests that there is no correlation between increased radial growth rate and conidial yield. The speed of germination was dictated by the protein content of the growth media as well as the size of the conidia, and this may explain why all of the tested strains displayed high stability and maximum germination up to $100 \%$ throughout successive subcultures. Notably, a positive relationship between the rate of conidial germination and virulence of the strain against the target pest was recorded by Dimbi et al. (2004) and Ansari and Butt (2011).

\section{Effect of successive subculturing on the activity of conidial Pr1 enzyme}

Following attachment to the insect cuticle, the conidia begin to germinate, exerting a combination of passive hydrophobic and electrostatic forces, followed by secretion of cuticle-degrading enzymes. One of the most important cuticle-degrading enzymes contribute to fungal pathogenicity is the subtilisin-like Pr1. Although Pr1 is not the only pathogenicity determinant for Metarhizium species, mutant $M$. anisopliae lacking the $\operatorname{Pr} 1$ gene was found to be less pathogenic (Wang et al., 2002). A slight fluctuation was reported in Pr1 production between ARSEF 4556, AUMC 3262, and V275 with nonsignificant differences through successive subcultures of 
Table 1 Effect of successive subculture on radial growth rate and conidial yield of ARSEF 4556, AUMC 3262 , and V275 after 15 days growth on SDAY at $25 \pm 2{ }^{\circ} \mathrm{C}$

\begin{tabular}{|c|c|c|c|c|c|c|}
\hline \multirow[t]{2}{*}{ Subcultures } & \multicolumn{3}{|c|}{ Radial growth rate (mm/day) } & \multicolumn{3}{|c|}{ Conidial yield $\left(\times 10^{7}\right)$} \\
\hline & ARSEF4556 & AUMC 3262 & V275 & ARSEF4556 & AUMC 3262 & V275 \\
\hline 1 st & $2.71 \pm 0.04^{\mathrm{a}}$ & $4.22 \pm 0.11^{a}$ & $3.39 \pm 0.54^{\mathrm{a}}$ & $6.23 \pm 0.23^{a}$ & $4.43 \pm 0.69^{\mathrm{a}}$ & $4.00 \pm 0.45^{a}$ \\
\hline 3 rd & $2.60 \pm 0.21^{a}$ & $4.13 \pm 0.0^{\mathrm{ab}}$ & $3.66 \pm 0.7^{\mathrm{a}}$ & $5.26 \pm 1.17^{b}$ & $2.46 \pm 0.38^{b}$ & $4.03 \pm 0.60^{a}$ \\
\hline 5 th & $2.75 \pm 0.08^{\mathrm{a}}$ & $4.08 \pm 0.04^{\mathrm{abc}}$ & $3.62 \pm 0.07^{\mathrm{a}}$ & $5.03 \pm 0.42^{b}$ & $1.93 \pm 0.40^{b}$ & $3.80 \pm 0.55^{a}$ \\
\hline 7th & $2.66 \pm 0.11^{\mathrm{a}}$ & $3.82 \pm 0.17^{\mathrm{bc}}$ & $3.77 \pm 0.02^{\mathrm{a}}$ & $3.70 \pm 0.15^{c}$ & $2.50 \pm 0.23^{b}$ & $4.46 \pm 0.38^{a}$ \\
\hline 9th & $2.60 \pm 0.06^{\mathrm{a}}$ & $3.73 \pm 0.13^{c}$ & $3.95 \pm 0.11^{\mathrm{a}}$ & $3.36 \pm 0.18^{c}$ & $2.70 \pm 0.15^{b}$ & $4.34 \pm 0.72^{\mathrm{a}}$ \\
\hline
\end{tabular}

Each value represents the mean of three replicates. The means within a column followed by the same letter are not significantly different (LDS test, $P>0.05$ )

the 3 Metarhizium strains $(P>0.05)$ (Table 2). Production of $\operatorname{Pr} 1$ by $M$. anisopliae and B. bassiana conidia is affected by different nutritional conditions as well as repeated subcultures (Dhar and Kaur, 2010 and Wanida and Poonsuk, 2012). In the same context, Shah et al. (2007) reported that spore adhesion, hydrophobicity, and spore-bound $\operatorname{Pr} 1$ declined, following the first subculture, as the spore-bound Pr1 was directly correlated with a decline in virulence, and so is used as a quality control marker to monitor changes in virulence. A slight fluctuation in Pr1 production between the examined strains and subcultures was noted, but this did not affect the virulence of the tested fungi. This is likely due to relative $\operatorname{Pr} 1$ production still being high enough to maintain a high virulence.

\section{Effect of successive subculture on conidial virulence}

Significant differences $(P<0.05)$ were noted among subcultures in the median lethal time $\left(\mathrm{LT}_{50}\right)$ against G. mellonella larvae. Table 2 shows the fluctuations in $\mathrm{LT}_{50}$ between the tested isolates, with conidia of V275 representing the most aggressive isolate with an $\mathrm{LT}_{50}$ of $1.69 \pm 0.12$ days with the conidia of the 1 st subculture. Conidia produced by AUMC 3262 subcultures displayed a high mortality in the range $1.92 \pm$ 0.03 to $2.34 \pm 0.09$ days, with 5 th and 9 th subcultures, respectively. Conidia of ARSEF 4556 were found to be less virulent than other strains with the highest
$\mathrm{LT}_{50}$ of $2.71 \pm 0.01$ days obtained with conidia produced by the 7th subculture. Nahar et al. (2008) and Santoro et al. (2014) recorded that M. anisopliae displayed a decreased virulence against Helicoverpa armigera Hubner (Lepidoptera: Noctuidae) after 2040 subcultures on nutrient-rich media. There is however little information about this phenomenon and how EPF stability changes when cultured on artificial media, but Brownbridge et al. (2001) believed the attenuation of virulence may be due to random mutation or caused by subculture conditions. Wang et al. (2005b) and Wang and St Leger (2005) studies on $M$. anisopliae returned this to the pathogenicity-related genes, which are upregulated differently when the fungus grows on different artificial media or media containing insect hemolymph.

\section{Conclusion}

EPF efficacy depends on several factors including fungal strain and the species of tested insects. Successive subculture affects greatly on the fungal quality control parameters, as appeared with both ARSEF4556 and AUMC3262, which showed changes in morphological characters, conidial yields and radial growth rates, and stability of both fungal viability and Pr1 production, while V275 displayed high stability with all examined parameters after repeated subculture.

Table 2 Influence of successive subculture on the activity of Pr1 enzyme and the LT 50 values of ARSEF4556, AUMC 3262, and V275 after 15 days growth on SDAY at $25 \pm 2^{\circ} \mathrm{C}$

\begin{tabular}{|c|c|c|c|c|c|c|}
\hline \multirow[t]{2}{*}{ Subcultures } & \multicolumn{3}{|c|}{$\operatorname{Pr} 1\left(\mu \mathrm{mol} \mathrm{ml} \mathrm{I}^{-1} \mathrm{~min}^{-1}\right)$} & \multicolumn{3}{|l|}{$\mathrm{LT}_{50}$} \\
\hline & ARSEF4556 & AUMC 2362 & V275 & ARSEF 4556 & AUMC3262 & V275 \\
\hline $1 \mathrm{st}$ & $0.73 \pm 0.015^{a}$ & $0.68 \pm 0.056^{a}$ & $0.77 \pm 0.025^{a}$ & $2.27 \pm 0.07^{b}$ & $2.14 \pm 0.04^{b}$ & $1.69 \pm 0.12^{c}$ \\
\hline 3rd & $0.72 \pm 0.013^{\mathrm{a}}$ & $0.73 \pm 0.030^{\mathrm{a}}$ & $0.77 \pm 0.016^{\mathrm{a}}$ & $2.34 \pm 0.09^{b}$ & $2.07 \pm 0.02^{b c}$ & $2.41 \pm 0.02^{\mathrm{a}}$ \\
\hline 5 th & $0.72 \pm 0.014^{\mathrm{a}}$ & $0.71 \pm 0.11^{\mathrm{a}}$ & $0.73 \pm 0.036^{\mathrm{a}}$ & $2.21 \pm 0.05^{b}$ & $1.92 \pm 0.03^{c}$ & $2.05 \pm 0.03^{b}$ \\
\hline 7th & $0.73 \pm 0.002^{\mathrm{a}}$ & $0.66 \pm 0.10^{\mathrm{a}}$ & $0.79 \pm 0.010^{a}$ & $2.71 \pm 0.01^{\mathrm{a}}$ & $2.21 \pm 0.05^{\mathrm{ab}}$ & $2.05 \pm 0.03^{b}$ \\
\hline 9th & $0.73 \pm 0.014^{\mathrm{a}}$ & $0.70 \pm 0.021^{\mathrm{a}}$ & $0.74 \pm 0.027^{a}$ & $2.36 \pm 0.02^{b}$ & $2.34 \pm 0.09^{a}$ & $2.27 \pm 0.07^{\mathrm{ab}}$ \\
\hline
\end{tabular}

Each value represents the mean of three replicates. The means within a column followed by the same letter are not significantly different (LSD test, $P>0.05$ ). $L T_{50}$ median lethal time. Pr1 activity is expressed as $\mu \mathrm{mol} \mathrm{ml}^{-1} \mathrm{~min}^{-1}$ released from succinyl-ala-ala-pro-phe-p-nitroanilide 


\section{Abbreviations}

AUMC: Assiut University Mycological Center; ARSEF: The Agricultural Research Service Collection of Entomopathogenic Fungal Cultures; IPM: Integrated pest management programs; Pr1: Protease enzyme; EPF: Entomopathogenic fungi; $\mathrm{LT}_{50}$ : Median lethal time

\section{Acknowledgements}

We are grateful to the Egyptian mission sector for their support of the first author in completing this research at Swansea University, UK. Also, we would like to express our sincere gratitude and appreciation to the biocontrol and natural products group at the department of biosciences, Swansea University, for their assistance and support.

\section{Authors' contributions}

TB suggested the aim of study and designed the experimental work. SE and $\mathrm{AE}$ designed the research and performed the statistical analysis. $\mathrm{RH}$ and JT conducted the study and wrote and revised the manuscript. All the authors read and approved the manuscript.

\section{Funding}

No fund.

\section{Availability of data and materials}

The datasets used and/or analyzed during the current study are available from the corresponding author on reasonable request.

\section{Ethics approval and consent to participate}

Not applicable.

\section{Consent for publication}

Not applicable.

\section{Competing interests}

The authors declare that they have no competing interests.

\section{Author details}

${ }^{1}$ Department of Botany, Faculty of Science, Zagazig University, Zagazig, Egypt. ${ }^{2}$ Department of Pest Physiology, Plant Protection Research Institute, Agricultural Research Center, Giza, Egypt. ${ }^{3}$ Department of Biosciences, Swansea University, Singleton Park, Swansea SA2 8PP, UK.

Received: 4 August 2020 Accepted: 6 December 2020

Published online: 02 January 2021

\section{References}

Ak K (2019) Efficacy of entomopathogenic fungi against the stored-grain pests, Sitophilus granarius L. and S. oryzae L. (Coleoptera: Curculionidae). Egypt J Biol Pest Control 29:12

Anand A, Tiwary BN (2009) Pathogenicity of entomopathogenic fungi to eggs and larvae of Spodoptera litura, the common cutworm. Biol Sci and Technol 19:919-929

Ansari MA, Butt TM (2011) Effects of successive subculturing on stability, virulence, conidial yield, germination, and shelf-life entomopathogenic fungi. J Appl Microbiol 110:1460-1469

Brownbridge M, Costa S, Jaronski ST (2001) Effects of invitro passage of Beauveria bassiana on virulence of Bemisia argentifolii. J Invertebr Pathol 77:280-283

Butt TM (2002) Use of entomopathogenic fungi for the control of insect pests. Mycota (Esser K \& Bennett JW. Eds), Volume editor Kempken, F. pp., 111-134.

Dhar P, Kaur G (2010) Production of cuticle degrading proteases by Beauveria bassiana and their induction in different media. Afr J Biochem Res 3:65-72

Dimbi S, Maniania NK, Lux SA, Mueke JM (2004) Effect of constant temperatures on germination, radial growth and virulence of Metarhizium anisopliae to three species of African tephritid fruit flies. Biocontrol 49:83-94

Ezzat SM, El-Sheikh AA, Hussien RHM (2019) Mass production of Metarhizium anisopliae AUMC 3262 strain isolated from Egyptian habitat and its virulence against Spodoptera littoralis larvae (Boisd.). Ann Agri Bio Res 24:277-282

Faria M, Wraight S (2007) Mycoinsecticides and mycoacaricides: a comprehensive list with worldwide coverage and international classification of formulation types. Biol Control 43:237-256

Firon A, Beauvais A, Latge JP, Grosjean C, Christoph E (2002) Characterization of essential genes by parasexual genetics in the human fungal pathogen
Aspergillus fumigatus: impact of genomic rearrangements associated with electroporation of DNA. Genetics 161:1077-1087

Han JH, Kim JJ, Lee SY (2014) Insecticidal activity of Metarhizium anisopliae FT83 against the different stages of beet armyworm, Spodoptera exigua. Kor J Pest Sci 18:417-421

Humber RA (2008) Evolution of entomopathogenicity in fungi. J Invertebr Pathol 98:262-266

Inglis GD, Enkerli J, Gottel MS (2012) Laboratory techniques used for entomopathogenic fungi: Hypocreales. In: Lacey LL (ed) Manual of techniques in invertebrate pathology. Academic Press, Amsterdam, pp 189253

Jenkins NE, Heviefo G, Langewald J, Cherry AJ, Lomer CJ (1998) Development of mass production technology for aerial conidia for use as mycopesticides. Biocontrol Inform 19:21-31

Nahar PB, Kulkarani SA, Kulye MS, Chavan SB, Kulkarani G, Rajendran A, Yadav PD, Shouche $Y$ (2008) Effect of repeated in vitro subculturing on the virulence of Metarhizium anisopliae against Helicoverpa armigera (Lepidoptera: Noctuidae). Biocontrol Sci Tech 18:337-355

Rayan MJ, Bridge PD, Smith D, Jeffries P (2002) Phyenogentic degradation occurs during sector formation in Metarhizium anisopliae. J Appl Microbiol 93, 163168

Safavi SA, Shah FA, Pakdel AK, Rasoulian GR, Bandani AR, Butt MT (2007) Effect of nutrition on growth and virulence of the entomopathogenic fungus Beauveria bassiana. FEMS Microbiol Lett 270:116-123

Santoro PH, Zorzetti J, Constanski K, Pedro MOJ (2014) Conidial production, virulence, and stress tolerance of Beauveria bassiana conidia after successive in vitro subculturing. Revista Colombiana de Entomología 40:85-90

Shah FA, Allen N, Wright CJ, Butt TM (2007) Repeated in-vitro subculturing alters spore surface properties and virulence of Metarhizium anisopliae. FEMS Microbiol Lett 276:60-66

Shah FA, Butt TM (2005) Influence of the nutrition on the production and physiology of sectors produced by the insect pathogenic fungus Metarhizium anisopliae. FEMS Microbiol Lett 250:201-207

Shah FA, Cheng SW, Butt MT (2005) Nutrition influences growth and virulence of the insect pathogenic fungus Metarhizium anisopliae. FEMS Microbiol Lett 251:259-266

Small CL, Bidochka MJ (2005) Up-regulation of Prl, a subtilisin like protease, during conidiation in the insect pathogen Metarhizium anisopliae. Mycol Res 109:307-313

St. Leger RJ, Charnley AK, Cooper RM (1987) Characterization of cuticle degrading proteases produced by the entomopathogen Metarhizium anisopliae. Arch Biochem Biophys 253:221-232

Wang C, Butt TM, St. Leger RJ (2005a) Colony sectorization of Metarhizium anisopliae is a sign of ageing. Microbiology 151:3223-3236

Wang C, Hu G, St. Leger RJ (2005b) Differential gene expression by Metarhizium anisopliae growing in roots exudates and host (Manduca sexta) cuticle or hemolymph reveals mechanisms of physiological adaptation. Fungal Genet Biol 42:704-718

Wang C, St Leger RJ (2005) Developmental and transcriptional responses to host and non-host cuticle by the specific locust pathogen Metarhizium anisopliae var. acridium. Eukaryot Cell 4:937-947

Wang C, Types MA, Butt TM (2002) Detection and characterization of pr1 virulent gene deficiencies in the insect pathogenic fungus Metarhizium anisopliae. FEMS Microbiol Lett 213:251-255

Wanida P, Poonsuk P (2012) Evaluation of strains of Metarhizium anisopliae and Beauveria bassiana against Spodoptera litura on the basis of their virulence, germination rate, conidia production, radial growth and enzyme activity. Mycobiology 40:111-116

\section{Publisher's Note}

Springer Nature remains neutral with regard to jurisdictional claims in published maps and institutional affiliations. 